B. Bongiorno and L. Di Piazza, Department of Mathematics, University of Palermo, Via Archirafi 34, 90123 Palermo, Italy. e-mail:

bongiorno@dipmat.math.unipa.it and dipiazza@dipmat.math.unipa.it

K. Musiał, Department of Mathematics, Wrocław University, Institute of Mathematics, Pl. Grunwaldzki 2/4, 50-384 Wrocław, Poland. e-mail:

musial@math.uni.wroc.pl

\title{
AN ALTERNATE APPROACH TO THE McSHANE INTEGRAL
}

\begin{abstract}
The classical McShane integral has been generalized by D.H. Fremlin [2] to the case of an arbitrary $\sigma$-finite outer regular quasi-Radon measure space. We present an alternate approach to the Fremlin integral for a non-atomic, finite quasi-Radon space.
\end{abstract}

\section{Introduction}

In [2] D.H. Fremlin studies, in a $\sigma$-finite outer regular quasi-Radon space, a method of integration for vector-valued functions which is a generalization of that from the McShane process of integration [5]. This method involves infinite McShane partitions by disjoint families of measurable sets of finite measure. However, in the compact case, the method may use finite McShane partitions with disjoint measurable sets. In the case of a compact space and a Radon measure, the use of suitable 'intervals', instead of measurable sets, is also possible: let $\mathcal{A}$ be an algebra of measurable sets such that whenever $F \subseteq G, F$ is closed and $G$ is open there is an $A \in \mathcal{A}$ such that $F \subseteq A \subseteq G$; the 'intervals' considered by Fremlin are the elements of a family $\mathcal{C} \subseteq \mathcal{A}$ such that every member of $\mathcal{A}$ is a finite disjoint union of members of $\mathcal{C}$.

In this paper, the context is a non-atomic, probability, and quasi-Radon space, $(\Omega, \mathcal{T}, \Sigma, \mu)$. We consider a method of integration which involves finite Henstock partitions by disjoint families of measurable sets that cover the whole domain up to a set of arbitrarily small measure (we call the partitions $\eta$-tight).

Key Words: vector valued function, McShane integral

Mathematical Reviews subject classification: Primary 28B05; Secondary 46G10

Received by the editors February 19, 1999 
We prove that it is equivalent to Fremlin's method. We may also consider $\eta$ tight partitions by disjoint families of 'intervals'. We have a larger choice in the definition of the 'intervals', indeed we may use semirings $\mathcal{S}$ satisfying the following weaker condition: given $\varepsilon>0, \omega \in \Omega$ and an open set $G$ of positive measure and containing $\omega$, there exists $A \in \mathcal{S}$ satisfying the condition: $\omega \in A \subseteq G$ and $\mu(G \backslash A)<\varepsilon$. In case the family $\mathcal{C}$ of 'intervals' is properly enclosed in $\mathcal{S}$ it may happen that no $\Delta$-fine Henstock $\eta$-tight partition exists, for some gauge $\Delta$ and sufficiently small $\eta$. In this case our method involves finite McShane $\eta$-tight partitions.

The use of finite McShane $\eta$-tight partitions may be applied to quite general families of sets $\mathcal{B}$. We do not know whether the $\mathcal{B}$-integral is always equivalent to the McShane integral considered by Fremlin. However, in Theorem 7 we prove this equivalence for real valued functions and in Theorem 9 for strongly measurable functions.

\section{Preliminaries}

Throughout $(\Omega, \mathcal{T}, \Sigma, \mu)$ will be a non-atomic, probability and quasi-Radon space (see [1]) i.e.:

(i) $(\Omega, \Sigma, \mu)$ is complete;

(ii) $\mathcal{T}$ is a $T_{1}$-topology such that $\mathcal{T} \subset \Sigma$;

(iii) $\mu(E)=\sup \{\mu(F): F \subseteq E, F$ is closed $\}$ for every $E \in \Sigma$;

(iv) $\mu$ is $\tau$-additive, i.e. if $\mathcal{G} \subseteq \mathcal{T}$ is non-empty and upwards directed by inclusion, then

$$
\mu\left(\bigcup_{G \in \mathcal{G}} G\right)=\sup \{\mu(G): G \in \mathcal{G}\} .
$$

The outer measure induced by $\mu$ is denoted by $\mu^{*}$. For $E \subset \Omega, \bar{E}, E^{c}$ and $\partial E$ are respectively the closure, the complement and the boundary of $E$.

Each function $\Delta: \Omega \rightarrow \mathcal{T}$ satisfying for each $\omega \in \Omega$ the property $\omega \in \Delta(\omega)$ will be called a gauge.

Let $\Delta$ be a gauge and let $E \subset \Omega$. Given a non-empty family $\mathcal{B} \subseteq \Sigma$, a collection $\mathcal{P}=\left\{\left(A_{n}, \omega_{n}\right): n=1, \ldots, p\right\}$ of pairwise disjoint sets $A_{n} \in \mathcal{B}$ and points $\omega_{n} \in A_{n}$ is said to be a Henstock $\mathcal{B}$-partition. If we assume only that $\omega_{n} \in \Omega, 1 \leq n \leq p$, then we say this is a McShane $\mathcal{B}$-partition. If there is no necessity to distinguish between them we will say simply about $\mathcal{B}$-partition. If all elements of the partition are subsets of $E$, then it is a $\mathcal{B}$-partition in $E$. A $\mathcal{B}$-partition is said to be: 
(a) tagged in $E$ if $\omega_{n} \in E$ for each $n \leq p$;

(b) $\Delta$-fine, if $A_{n} \subset \Delta\left(\omega_{n}\right)$ for each $n \leq p$;

(c) $\eta$-tight, if $\mu\left(\Omega \backslash \bigcup_{n=1}^{p} A_{n}\right)<\eta$, where $\eta$ is a positive number.

Given a partition $\mathcal{P}=\left\{\left(A_{n}, \omega_{n}\right): n=1, \ldots, p\right\}$, we set $\operatorname{co}(\mathcal{P})=\cup_{n} A_{n}$. For simplicity, a $\mathcal{B}$-partition that is $\Delta$-fine and $\eta$-tight will be called a $(\Delta, \eta, \mathcal{B})$ partition. If $\mathcal{P}$ is a $\Delta$-fine $\mathcal{B}$-partition in $E$, and $\mu[E \backslash \operatorname{co}(\mathcal{P})]<\eta$, then it is called a $(\Delta, \eta, \mathcal{B})$-partition of $E$. Whenever the set where the partition is tagged is not specified, then it means that the partition is tagged in $\Omega$. If $\mathcal{B} \subseteq \Sigma$, we denote by $\mathcal{B}^{\cup}$ the family of all finite unions of members of $\mathcal{B}$. We say that $\mathcal{T}$ is inner regular with respect to $\mathcal{B}$ whenever $\mu(G)=\sup \{\mu(B): \mathcal{B} \ni$ $B \subseteq G\}$, for each $G \in \mathcal{T}$.

The following result may be considered as a partial justification of our further assumptions concerning $\mathcal{B}$ :

Proposition 1. Let $\mathcal{B} \subseteq \Sigma$ be a collection of sets and let $\mathcal{C} \subset \mathcal{B}$ be such that each member of $\mathcal{B}$ is a finite union of pairwise disjoint elements of $\mathcal{C}$. If $\mathcal{T}$ is inner regular with respect to $\mathcal{B}$, then for each $G \in \mathcal{T}$ the set of all $\omega \in G$ such that for each $\varepsilon>0$ there exists $G \supseteq C \in \mathcal{C}$ of positive measure less than $\varepsilon$ and containing the point $\omega$ is of full measure in $G$.

Proof. Since $\mu$ is nonatomic, for each $n \in \mathbf{N}$ there exists a decomposition $G=\cup_{i=1}^{n} H_{i}^{n}$ into pairwise disjoint sets of measure $\mu(G) / n$. Because of the outer regularity of $\mu$ for each $i \leq n$ there is an open set $G_{i}^{n} \supset H_{i}^{n}$, such that $G_{i}^{n} \subset G$ and $\mu\left(G_{i}^{n} \backslash H_{i}^{n}\right)<\mu(G) / n 2^{n}$. It follows from the inner regularity of $\mathcal{T}$, that for each $i \leq n$ one can find $B_{i}^{n} \in \mathcal{B}$ with $\mu\left(G_{i}^{n} \backslash B_{i}^{n}\right)<\mu(G) / n 2^{n}$. Consequently,

$$
\mu\left(G \backslash \bigcup_{i=1}^{n} B_{i}^{n}\right) \leq \mu\left(\bigcup_{i=1}^{n}\left(H_{i}^{n} \backslash B_{i}^{n}\right)\right) \leq \sum_{i=1}^{n} \mu\left(G_{i}^{n} \backslash B_{i}^{n}\right)<\mu(G) / 2^{n} .
$$

If $B_{i}^{n}=\cup_{j=1}^{k(i, n)} C_{j}^{n, i}$ with $C_{j}^{n, i} \in \mathcal{C}$, then some of the sets $C_{j}^{n, i}$ may be of measure zero; denote their union by $N_{i}^{n}$.

For each $n$ put $H_{n}=: \bigcup_{i=1}^{n} B_{i}^{n} \backslash \bigcup_{i=1}^{n} N_{i}^{n}$. Then $\mu\left(H_{n}^{c}\right)<\mu(G) / 2^{n}$. Consider now the set $\lim \sup _{n} H_{n}$. If $\omega \in \lim \sup _{n} H_{n}$, then for each $\varepsilon>0$ there exists an element of $\mathcal{C}$ containing $\omega$ and of measure less than $\varepsilon$. Now we have :

$$
\mu\left(G \backslash \limsup _{n} H_{n}\right)=\mu\left(\bigcup_{n=1}^{\infty} \bigcap_{k=n}^{\infty} H_{k}^{c}\right)=\lim _{n} \mu\left(\bigcap_{k=n}^{\infty} H_{k}^{c}\right)=0 .
$$

This completes the proof. 
Following the above lemma, we say that a family $\mathcal{B}$ is fine on $\Omega$ if for all $\omega \in \Omega$ the following is satisfied: for each $G \in \mathcal{T}$ with $\omega \in G$ and $\mu(G)>0$, there exists $B \in \mathcal{B}$ such that $\omega \in B \subset G$ and $\mu(B)>0$. It is clear that together with outer regularity of $\mu$ this means that each point $\omega \in \Omega$ can be embedded into a member of $\mathcal{B}$ of arbitrary small measure. We say that $\mathcal{B} \subseteq \Sigma$ separates points off closed sets if given $\varepsilon>0, \omega \in \Omega$ and an open $G$ of positive measure and containing $\omega$, there exists $B \in \mathcal{B}$ satisfying the condition $\omega \in B \subseteq G$ and $\mu(G \backslash B)<\varepsilon$.

From now on we assume that $\mathcal{S} \subseteq \Sigma$ is a semiring of sets, i.e.

$$
\text { if } A, B \in \mathcal{S} \text { then } A \cap B \in \mathcal{S} \text { and } A \backslash B \in \mathcal{S} \text {. }
$$

Throughout $\mathcal{C} \subseteq \mathcal{S}$ is a fine collection of sets, such that each $R \in \mathcal{S}$ is a finite union of pairwise disjoint members of $\mathcal{C} . W e$ assume also that $\mathcal{S}$ separates points off closed sets.

It follows that $\mathcal{T}$ is inner regular with respect to $\mathcal{S}$ (or equivalently: $\mathcal{S}$-regular).

Remark 1. In [2] Proposition $1 \mathrm{~F}$, Fremlin considers an algebra $\mathcal{A}$ satisfying the following stronger condition:

given a closed set $F$ and an open $G$ such that $F \subseteq G$, there exists $A \in \mathcal{A}$ with

$$
F \subseteq A \subseteq G .
$$

Notice that an algebra separating points off closed sets not necessarily satisfies the above condition. Indeed let us consider the following two examples:

Example 1. Let $\mathcal{A}$ be the algebra generated by intervals of $\mathbf{R}$ and let $\mu$ be a Gaussian measure on $\mathbf{R}$. Then $\mathcal{A}$ separates points off closed sets, but does not satisfy Fremlin's condition.

In fact let $F=\mathbf{N}$ and let $G=\bigcup_{n \in \mathbf{N}}\left(n-\frac{1}{2}, n+\frac{1}{2}\right)$. It is impossible to find such $A$ since it should be a union of finitely many intervals only.

Example 2. Let $\mathcal{B}$ be the family of first category subsets of $[0,1]$ and let $\mu$ be the Lebesgue measure. According to a theorem of Marczewski and Sikorski [4] if $G$ is an open subset of [0,1], then $G$ can be decomposed into disjoint sets $K$ and $M$ such that $K \in \mathcal{B}$ and $\mu(M)=0$. It follows that the ring $\mathcal{B}$ separates points off closed sets. Indeed, if $\omega \in G$ then we simply set $B=K \cup\{\omega\}$. On the other hand, since the algebra $\mathcal{A}$ generated by $\mathcal{B}$ consists of sets which are of the first category or their complements are such, it cannot satisfy Fremlin's property. Indeed, if we take $F=[1 / 3,1 / 2]$ and $G=(1 / 4,3 / 4)$, then each $A \in \mathcal{A}$ such that $F \subseteq A \subseteq G$ is of the second category and its complement $A^{c}$ either. Thus, $A$ cannot be a member of the algebra generated by $\mathcal{B}$. 


\section{$3 \quad \mathcal{B}$-Integrals Generated by Semirings and Intervals.}

One of the most essential problems in the theory of gauge integrals is the existence of $\Delta$-fine partitions. In the case of partitions generated by a semiring or by a family $\mathcal{C}$ of intervals the following result holds:

Lemma 1. If $W \subseteq \Omega$ is such that $\mu^{*}(W)=1$, then for each $\eta>0$ and each gauge $\Delta$ there exists a $(\Delta, \eta, \mathcal{C})$-McShane partition tagged in $W$ and $a$ $(\Delta, \eta, \mathcal{S})$-Henstock partition tagged in $W$.

Proof. Let $\eta>0$ and a gauge $\Delta: \Omega \rightarrow \mathcal{T}$ be given. Then $\Omega \subseteq \bigcup_{\omega \in \Omega} \Delta(\omega)$. Let us fix an arbitrary $\omega_{0} \in W$ such that $\mu\left(\Delta\left(\omega_{0}\right)\right)>0$ (such a point exists because $\mu$ is $\tau$-additive). Assume now that $\kappa$ is the first ordinal of the cardinality equal to the cardinality of the set $\Omega$ and that for some $1 \leq \gamma<\kappa$ we already have a collection $\left\{\omega_{\alpha} \in W: \alpha<\gamma\right\}$ such that :

(i) $\mu\left(\Delta\left(\omega_{\alpha}\right)\right)>0$ for each $\alpha<\gamma$;

(ii) $\omega_{\beta} \notin \Delta\left(\omega_{\alpha}\right)$ for each $\alpha<\beta<\gamma$.

If $\mu\left[\bigcup_{\alpha<\gamma} \Delta\left(\omega_{\alpha}\right)\right]=1$, then we finish the construction. But if $\mu\left[\bigcup_{\alpha<\gamma} \Delta\left(\omega_{\alpha}\right)\right]<$ 1 , then we have the inclusion

$$
\Omega_{\gamma} \subseteq \bigcup_{\omega \in \Omega_{\gamma}} \Delta(\omega)
$$

where,for simplicity we put $\Omega_{\gamma}=\Omega \backslash \bigcup_{\alpha<\gamma} \Delta\left(\omega_{\alpha}\right)$, and the set $\Omega_{\gamma}$ is of positive measure. Since the measure $\mu$ restricted to $\Omega_{\gamma}$ is also $\tau$-additive, there exists a point $\omega_{\gamma} \in \Omega_{\gamma} \cap W$ such that $\mu\left[\Delta\left(\omega_{\gamma}\right) \cap \Omega_{\gamma}\right]>0$.

Since $\mu$ is finite, it satisfies the CCC condition (i.e. each pairwise disjoint family of sets of positive measure is at most countable) and so there is a countable ordinal $\gamma<\kappa$ such that $\mu\left(\Omega_{\gamma}\right)=0$. Applying once again the $\tau$ additivity of $\mu$, we find a finite collection $\bar{\omega}_{1}, \ldots, \bar{\omega}_{n} \in\left\{\omega_{\alpha}: \alpha<\gamma\right\}$ such that

$$
a:=\mu\left(\Omega \backslash \bigcup_{i=1}^{n} \Delta\left(\bar{\omega}_{i}\right)\right)<\eta .
$$

Now by the properties of the family $\mathcal{S}$, we can find for each $i \in\{1, \ldots, n\}$ a set $B_{i} \in \mathcal{S}$ such that $\bar{\omega}_{i} \in B_{i} \subseteq \Delta\left(\bar{\omega}_{i}\right)$ and $\mu\left[\Delta\left(\bar{\omega}_{i}\right) \backslash B_{i}\right]<(\eta-a) / n$. It follows that

$$
\mu\left(\bigcup_{i=1}^{n} \Delta\left(\bar{\omega}_{i}\right) \backslash \bigcup_{i=1}^{n} B_{i}\right) \leq \mu\left(\bigcup_{i=1}^{n}\left[\Delta\left(\bar{\omega}_{i}\right) \backslash B_{i}\right]\right) \leq \sum_{i=1}^{n} \mu\left[\Delta\left(\bar{\omega}_{i}\right) \backslash B_{i}\right]<\eta-a .
$$


Thus,

$$
\mu\left(\Omega \backslash \bigcup_{i=1}^{n} B_{i}\right) \leq \mu\left(\Omega \backslash \bigcup_{i=1}^{n} \Delta\left(\bar{\omega}_{i}\right)\right)+\mu\left(\bigcup_{i=1}^{n} \Delta\left(\bar{\omega}_{i}\right) \backslash \bigcup_{i=1}^{n} B_{i}\right)<\eta .
$$

Setting $R_{1}=B_{1}, \ldots, R_{n}=B_{n} \backslash \bigcup_{i=1}^{n-1} B_{i}=\left(\cdots\left(B_{n} \backslash B_{1}\right) \backslash \cdots \backslash B_{n-1}\right)$, we get a $(\Delta, \eta, \mathcal{S})$-Henstock partition $\left\{\left(R_{i}, \bar{\omega}_{i}\right): i \leq p\right\}$, that is tagged in $W$. By the assumption however each $R_{i}$ is of the form $R_{i}=\bigcup_{j=1}^{k_{i}} C_{i}^{j}, i \leq n$. $\left\{\left(C_{i}^{j}, \bar{\omega}_{i}\right): j \leq k_{i}, i \leq n\right\}$ is the required McShane $(\Delta, \eta, \mathcal{C})$-partition tagged in $W$.

Let $X$ be a Banach space and let $f: \Omega \rightarrow X$. For each partition $\mathcal{P}=$ $\left\{\left(A_{n}, \omega_{n}\right): n=1, \ldots, p\right\}$, we set $\sigma(f, \mathcal{P})=\sum_{n=1}^{p} f\left(\omega_{n}\right) \mu\left(A_{n}\right)$.

Definition 1. Let $\mathcal{B} \subseteq \Sigma$ be an arbitrary collection of sets such that for every $\eta>0$ and every gauge $\Delta$ there exists a $(\Delta, \eta, \mathcal{B})$-McShane (resp. Henstock) partition. We say that $f$ is $\mathcal{B}_{M S}$ (resp. $\mathcal{B}_{H}$ ) -integrable on $\Omega$ if there exists $w \in X$ satisfying the following property:

for each $\varepsilon>0$ there exists a gauge $\Delta: \Omega \rightarrow \mathcal{T}$ and a positive constant $\eta$ such that

$$
\|\sigma(f, \mathcal{P})-w\|<\varepsilon
$$

for every $(\Delta, \eta, \mathcal{B})$-McShane (resp. Henstock) partition $\mathcal{P}$.

We set $w=\left(\mathcal{B}_{M S}\right) \int_{\Omega} f d \mu$ and $w=\left(\mathcal{B}_{H}\right) \int_{\Omega} f d \mu$ respectively.

Given a measurable set $E \subset \Omega$ it is said that $f$ is $\mathcal{B}_{M S}$ (resp. $\mathcal{B}_{H}$ ) -integrable on $E$ if the function $f \chi_{E}$ is $\mathcal{B}_{M S}$ (resp. $\left.\mathcal{B}_{H}\right)$-integrable on $\Omega$. We set $w(E)=$ $\left(\mathcal{B}_{M S}\right) \int_{\Omega} f \chi_{E} d \mu$ and $w(E)=\left(\mathcal{B}_{H}\right) \int_{\Omega} f \chi_{E} d \mu$ respectively.

It is clear that without the assumption concerning the existence of $(\Delta, \eta, \mathcal{B})$ partitions the above integrals may not exist. In case of $\mathcal{B}=\mathcal{C}$ or $\mathcal{B}=\mathcal{S}$ the $\mathcal{B}$-integrals are well defined, that is each function has at most one value of the integral. Indeed, suppose there exist $w_{i} \in X$, a gauge $\Delta_{i}: \Omega \rightarrow \mathcal{T}$ and $\eta_{i}>0$ such that $\left\|\sigma\left(f, \mathcal{P}_{i}\right)-w_{i}\right\|<\varepsilon$ for each $\left(\Delta_{i}, \eta_{i}, \mathcal{B}\right)$-McShane partition $\mathcal{P}_{i}, i=1,2$. Then define $\Delta(\omega)=\Delta_{1}(\omega) \cap \Delta_{2}(\omega), \omega \in \Omega$ and $\eta=\min \left\{\eta_{1}, \eta_{2}\right\}$. Let $\mathcal{P}$ be a $(\Delta, \eta, \mathcal{B})$-McShane partition (existing in virtue of Lemma 1$)$. We have

$$
\left\|w_{1}-w_{2}\right\| \leq\left\|\sigma(f, \mathcal{P})-w_{1}\right\|+\left\|\sigma(f, \mathcal{P})-w_{2}\right\|<2 \varepsilon .
$$

Thus $w_{1}=w_{2}$. In the same way, one proves the uniqueness of the $\mathcal{B}_{H}$-integral.

Remark 2. If $f$ is $\Sigma$-McShane $\mathcal{B}$-integrable, then it is $\mathcal{B}_{M S}$-integrable for quite arbitrary $\mathcal{B} \subset \Sigma$ (such that the definition makes sense). It is clear that the opposite implication is in general false, since $\mathcal{B}$ can be very poor. A similar fact holds true also for the Henstock version. 
Lemma 2. Let $f: \Omega \rightarrow X$ be a function almost everywhere equal to zero on $\Omega$. Then $f$ is $\mathcal{S}_{M S}$ (resp. $\mathcal{S}_{H}$ ) -integrable with $\mathcal{S}_{M S}$ (resp. $\mathcal{S}_{H}$ ) -integral equal to zero. The same result holds true for $\mathcal{C}_{M S}$-integral.

Proof. For each $n=1,2 \ldots$, let $N_{n}=\{\omega \in \Omega: n-1<\|f(\omega)\| \leq n\}$. It follows that $\mu\left(\cup_{n} N_{n}\right)=0$. Fix $\varepsilon>0$, take $0<\eta<\varepsilon$ and for each $n \in \mathbf{N}$, choose an open set $G_{n}$ such that $G_{n} \supset N_{n}$ and $\mu\left(G_{n}\right)<\eta / n 2^{n}$.

Then define $\Delta: \Omega \rightarrow \mathcal{T}$ so that $\Delta(\omega)=G_{n}$, if $\omega \in N_{n}$. If $\mathcal{P}=\left\{\left(A_{n}, \omega_{n}\right)\right.$ : $n=1, \ldots, p\}$ is a $(\Delta, \eta, \mathcal{A})$-McShane partition, then

$$
\|\sigma(f, \mathcal{P})\|=\left\|\sum_{n} \sum_{\omega_{i} \in N_{n}} f\left(\omega_{i}\right) \mu\left(A_{i}\right)\right\| \leq \sum_{n} n \sum_{\omega_{i} \in N_{n}} \mu\left(A_{i}\right)<\eta \sum_{n} 2^{-n}<\varepsilon .
$$

Definition 2. It is said that $f$ is Fremlin-integrable on $\Omega$ (see Fremlin [2]) if there exists $w \in X$ satisfying the following property:

for each $\varepsilon>0$ there exists a gauge $\Delta$ such that

$$
\limsup _{k \rightarrow \infty}\left\|\sum_{n=1}^{k} f\left(x_{n}\right) \mu\left(A_{n}\right)-w\right\|<\varepsilon,
$$

for every $\Delta$-fine infinite McShane $\Sigma$-partition $\left\{\left(A_{n}, x_{n}\right): n \in \mathbf{N}\right\}$ such that $\mu\left(\Omega \backslash \cup_{n=1}^{\infty} A_{n}\right)=0$. We set $w=(F) \int_{\Omega} f d \mu$.

Theorem 3. A function $f: \Omega \rightarrow X$ is $\Sigma_{M S}$-integrable on $\Omega$ if and only if it is $\Sigma_{H}$-integrable on $\Omega$, and if and only if it is Fremlin-integrable on $\Omega$.

Proof. The first equivalence is obvious. Let now $f$ be $\Sigma_{M S}$-integrable on $\Omega$. Fix $\varepsilon>0$ and let $\Delta, \eta$ and $w$ be such that

$$
\|\sigma(f, \mathcal{P})-w\|<\varepsilon,
$$

for each $(\Delta, \eta, \Sigma)$-McShane partition $\mathcal{P}$.

Now let $\mathcal{Q}=\left\{\left(A_{i}, \omega_{i}\right): i \in \mathbf{N}\right\}$ be an infinite $\Delta$-fine McShane partition such that $\sum_{i=1}^{\infty} \mu\left(A_{i}\right)=\mu(\Omega)$. Let $i_{0}$ be such that $\sum_{i>i_{0}} \mu\left(A_{i}\right)<\eta$. Note that $\left\{\left(\left(A_{i} \backslash \cup_{j=1}^{\infty}\left\{\omega_{j}\right\}\right) \cup\left\{\omega_{i}\right\}, \omega_{i}\right): i \leq j\right\}$ is a $(\Delta, \eta, \Sigma)$-partition for every $j>i_{0}$. Then we have for every $j>i_{0}$

$$
\left\|\sum_{i \leq j} f\left(\omega_{i}\right) \mu\left(A_{i}\right)-w\right\|<\varepsilon
$$

Hence $f$ is Fremlin-integrable. 
If $f$ is Fremlin-integrable on $\Omega$, then it is Pettis integrable and the integrals coincide (see [2], Theorem 1Q). Since the indefinite Pettis integral is absolutely continuous with respect $\mu$, for each $\varepsilon>0$ we can find a positive constant $\eta$ such that

$$
\left\|(F) \int_{E} f d \mu\right\|<\frac{\varepsilon}{2}, \quad \text { whenever } \mu(E)<\eta .
$$

Moreover, by [2] (Lemma 2B) we can find a gauge $\Delta$ such that

$$
\left\|\sigma(f, \mathcal{P})-(F) \int_{c o(\mathcal{P})} f d \mu\right\|<\frac{\varepsilon}{2},
$$

whenever $\mathcal{P}$ is a McShane $\Delta$-fine $\Sigma$-partition.

Thus, if $\mathcal{P}$ is a $(\Delta, \eta, \Sigma)$-McShane partition, we have:

$$
\begin{aligned}
& \left\|\sigma(f, \mathcal{P})-(F) \int_{\Omega} f d \mu\right\| \\
& \leq\left\|\sigma(f, \mathcal{P})-(F) \int_{c o(\mathcal{P})} f d \mu\right\|+\left\|(F) \int_{\Omega \backslash c o(\mathcal{P})} f d \mu\right\| \\
& <\frac{\varepsilon}{2}+\frac{\varepsilon}{2}=\varepsilon .
\end{aligned}
$$

Theorem 4. Assume that $\mathcal{C} \subset \mathcal{S}$ is fine and $\mathcal{S}$ separates points off closed sets. For an arbitrary function $f: \Omega \rightarrow X$ the following are equivalent:

(i) $f$ is $\Sigma_{M S}$-integrable on $\Omega$;

(ii) $f$ is $\mathcal{C}_{M S}$-integrable on $\Omega$;

(iii) $f$ is $\mathcal{S}_{H}$-integrable on $\Omega$.

Proof. It follows directly by definitions that if $f$ is $\Sigma_{M S}$-integrable on $\Omega$ then it is $\mathcal{S}_{H}$-integrable on $\Omega$ and - in the same way - it is $\mathcal{C}_{M S}$-integrable. Moreover the integrals coincide.

We will prove now, that $\mathcal{C}_{M S}$-integrability yields $\mathcal{S}_{H}$-integrability. To see it assume that $f$ is $\mathcal{C}_{M S}$-integrable. Then fix $\varepsilon>0$ and take $\eta>0$ and a gauge $\Delta$ such that

$$
\left\|\sigma(f, \mathcal{Q})-\left(\mathcal{C}_{M S}\right) \int_{\Omega} f d \mu\right\|<\varepsilon
$$

for each $(\Delta, \eta, \mathcal{C})$-McShane partition $\mathcal{Q}=\left\{\left(C_{i}, w_{i}\right): i=1, \ldots, s\right\}$. Let $\mathcal{P}=$ $\left\{\left(A_{i}, \omega_{i}\right): i=1, \ldots, p\right\}$ be a $(\Delta, \eta, \mathcal{S})$-Henstock partition. It follows from the 
assumed properties of $\mathcal{C}$, that for each $i \leq p$ we have $A_{i}=\bigcup_{j=1}^{k_{i}} C_{i}^{j}$, where $C_{i}^{1}, \ldots, C_{i}^{k_{i}}$ are pairwise disjoint members of $\mathcal{C}$. If we set $\mathcal{V}:=\left\{\left(C_{i}^{j}, \omega_{i}\right): j \leq\right.$ $\left.k_{i}, i \leq p\right\}$, then it follows from the inequality (4) that

$$
\left\|\sigma(f, \mathcal{V})-\left(\mathcal{S}_{H}\right) \int_{\Omega} f d \mu\right\|<\varepsilon
$$

what proves the $\mathcal{S}_{H}$-integrability of $f$.

Now suppose $f$ is $\mathcal{S}_{H}$-integrable on $\Omega$. We are going to show that $f$ is $\Sigma_{H}$-integrable. Fix $\varepsilon>0$ and take $0<\eta<\varepsilon / 8$ and a gauge $\Delta$ such that

$$
\left\|\sigma(f, \mathcal{Q})-\left(\mathcal{S}_{H}\right) \int_{\Omega} f d \mu\right\|<\frac{\varepsilon}{2}
$$

for each $(\Delta, \eta, \mathcal{S})$-Henstock partition $\mathcal{Q}=\left\{\left(A_{i}, w_{i}\right): i=1, \ldots, s\right\}$. Let $\mathcal{P}=\left\{\left(E_{i}, \omega_{i}\right): i=1, \ldots, p\right\}$ be a $(\Delta, \eta, \Sigma)$-Henstock partition. Put $m:=$ $\max _{i}\left\|f\left(\omega_{i}\right)\right\|$. The proof will be inductive. Assume that for some $1 \leq q<p$ we have already sets $A_{1}, \ldots, A_{q} \in \mathcal{S}$, such that $\omega_{j} \in A_{j}$ for $j \leq q$, closed sets $F_{1}, \ldots, F_{q}$ and open sets $G_{1}, \ldots, G_{q}$ satisfying for each $j \leq q$ the following properties:

$$
\begin{gathered}
F_{j} \subseteq A_{j} \subseteq G_{j} \quad \text { and } \quad E_{j} \subseteq G_{j} \subseteq \Delta\left(\omega_{j}\right) ; \\
G_{i} \cap\left(\left\{\omega_{1}, \ldots, \omega_{p}\right\} \backslash\left\{\omega_{i}\right\}\right)=\emptyset \quad \text { and } \quad G_{i} \cap F_{j}=\emptyset \quad \text { if } j<i \leq q ; \\
\mu\left(G_{j} \backslash F_{j}\right)<\frac{2 \eta}{p^{2}(m+1)} \quad \text { and } \quad \mu\left(E_{j} \triangle A_{j}\right)<\frac{2 \eta}{p^{2}(m+1)} .
\end{gathered}
$$

Having these sets, we take an open set $G_{q+1}$ such that

$$
E_{q+1} \subseteq G_{q+1} \subseteq \Delta\left(\omega_{q+1}\right), \quad G_{q+1} \cap\left(\left\{\omega_{1}, \ldots, \omega_{q}\right\} \backslash\left\{\omega_{q+1}\right\}\right)=\emptyset
$$

and

$$
\mu\left(G_{q+1} \backslash E_{q+1}\right)<\frac{\eta}{p^{2}(m+1)}
$$

(besides the outer regularity of $\mu$ we apply here the $T_{1}$-property of $\mathcal{T}$ and the non-atomicity of $\mu)$. Since $E_{q+1} \cap\left(\bigcup_{j \leq q} E_{j}\right)=\emptyset$, we may take $G_{q+1}$ in such a way that

$$
G_{q+1} \cap F_{j}=\emptyset \quad \text { if } \quad j \leq q .
$$


By the properties of $\mathcal{S}$, there exists $A_{q+1} \in \mathcal{S}$, such that

$$
\omega_{q+1} \in A_{q+1} \subseteq G_{q+1} \quad \text { and } \quad \mu\left(G_{q+1} \backslash A_{q+1}\right)<\frac{\eta}{p^{2}(m+1)} .
$$

Then, by the inner regularity of $\mu$, we take a closed set $F_{q+1} \subseteq A_{q+1}$ such that

$$
\mu\left(A_{q+1} \backslash F_{q+1}\right)<\frac{\eta}{p^{2}(m+1)} .
$$

Then we have

$$
\mu\left(G_{q+1} \backslash F_{q+1}\right)<\frac{2 \eta}{p^{2}(m+1)} \quad \text { and } \quad \mu\left(E_{q+1} \triangle A_{q+1}\right)<\frac{2 \eta}{p^{2}(m+1)} .
$$

Since the first step is similar to the inductive one (we set $F_{0}=\emptyset$ in $(6)$ ), the construction is over.

By (6) and (7) we deduce that, for each $i, j \leq p, \omega_{i} \in A_{i}$ and $\omega_{j} \notin A_{i}$ if $j \neq i$. Now put $B_{1}=A_{1}, B_{2}=A_{2} \backslash A_{1}, \ldots, B_{p}=A_{p} \backslash\left(\cup_{j=1}^{p-1} A_{j}\right)=$ $\left(\cdots\left(A_{p} \backslash A_{1}\right) \backslash \cdots \backslash A_{p-1}\right)$. By the construction $\left\{\left(B_{i}, \omega_{i}\right): i=1, \ldots, p\right\}$ is a $(\Delta, \eta, \mathcal{S})$-Henstock partition. Then notice that, from the relations $A_{j} \subseteq G_{j}$ and $G_{i} \cap F_{j}=\emptyset$ for $j<i$, it follows

$$
E_{i} \cap A_{j} \subseteq E_{i} \cap G_{j} \subseteq G_{i} \cap G_{j} \subseteq G_{j} \backslash F_{j} \quad \text { if } \quad j<i \leq p .
$$

Hence, we have

$$
\begin{aligned}
B_{i} \triangle E_{i} & =\left(E_{i} \backslash B_{i}\right) \cup\left(B_{i} \backslash E_{i}\right) \subseteq\left(\cup_{j<i} E_{i} \cap A_{j}\right) \cup\left(A_{i} \triangle E_{i}\right) \\
& \subseteq \cup_{j<i}\left(G_{j} \backslash F_{j}\right) \cup\left(A_{i} \triangle E_{i}\right) .
\end{aligned}
$$

So by (8) it results

$$
\mu\left(B_{i} \triangle E_{i}\right) \leq \mu\left(A_{i} \triangle E_{i}\right)+\sum_{j<i} \mu\left(G_{j} \backslash F_{j}\right)<\frac{4 \eta}{p(m+1)} .
$$

Thus by (5) and by (9) we obtain

$$
\begin{aligned}
& \left\|\sum_{i=1}^{p} f\left(\omega_{i}\right) \mu\left(E_{i}\right)-\left(\mathcal{S}_{H}\right) \int_{\Omega} f d \mu\right\| \\
& \leq\left\|\sum_{i=1}^{p} f\left(\omega_{i}\right) \mu\left(B_{i}\right)-\left(\mathcal{S}_{H}\right) \int_{\Omega} f d \mu\right\|+\sum_{i=1}^{p}\left\|f\left(\omega_{i}\right)\right\| \mu\left(B_{i} \triangle E_{i}\right) \\
& <\varepsilon / 2+4 \eta<\varepsilon .
\end{aligned}
$$

In virtue of Theorem $3 f$ is $\Sigma_{M S}$-integrable. 


\section{$4 \mathcal{B}$-Integrals Generated by Arbitrary Families of Sets.}

We noticed earlier that we need to add some hypotheses either on the family $\mathcal{B} \subset \Sigma$ or on $\Omega$ to guarantee the existence of a $\Delta$-fine $\eta$-tight partition. We are now going to present a proper example.

Example 3. Let $0<\eta<1$ and let us consider $\Omega=[0,1]$ endowed with the usual topology and the Lebesgue measure $\mu$. Fix two decreasing sequences of positive numbers $\left(r_{n}\right),\left(R_{n}\right)$ such that $0<r_{n}<R_{n}<r_{n-1}<1 / 4$ and such that

$$
\sum_{n=1}^{\infty} \frac{r_{n}}{r_{n}+R_{n}}<1-\eta
$$

Define a sequence $\left(Q_{n}\right)$ of finite disjoint sets such that $Q_{n} \subset[0,1]$ and every interval of length $R_{n}$ meets $Q_{n}$. Then define $\mathcal{B}_{0}$ as the family of all finite subsets of $[0,1]$ and by $\mathcal{B}_{n}, n \geq 1$, the family of all sets $B$ such that there is an interval $I \subset[0,1]$ and a set $F \in \mathcal{B}_{0}$ with the following property:

$$
r_{n+1} \leq \mu(I)<r_{n}
$$

and

$$
B=(I \backslash F) \cup\left\{x \in Q_{n}: \operatorname{dist}(x, I)<4 R_{n}\right\} .
$$

Set $\mathcal{B}=\bigcup_{n=0}^{\infty} \mathcal{B}_{n}$.

Then, if $\left\{B_{k}, k=1,2, \cdots\right\}$ is a family of disjoint $\mathcal{B}$-sets, we have:

$$
\sum_{k=1}^{\infty} \mu\left(B_{k}\right)=\sum_{n} \sum_{B_{k} \in \mathcal{B}_{n}} \mu\left(B_{k}\right) .
$$

Now, for each $n>1$ we can have at most $\left(r_{n+1}+3 R_{n}\right)^{-1}$ elements of $\left\{B_{k}\right\}$ belonging to the family $\mathcal{B}_{n}$. Moreover

$$
r_{n}-r_{n+1}<R_{n}-r_{n+1}<R_{n}<2 R_{n}
$$

and

$$
r_{n}+R_{n}<r_{n+1}+3 R_{n}
$$

Then

$$
\sum_{k=1}^{\infty} \mu\left(B_{k}\right) \leq \sum_{n=1}^{\infty} \frac{r_{n}}{r_{n}+R_{n}}<1-\eta .
$$

Thus, for any gauge $\Delta$ no $(\Delta, \eta, \mathcal{B})$-partition exists (neither Henstock nor McShane). Notice however that, since $\left(R_{n}\right)$ is decreasing to zero, the family $\mathcal{B}$ is fine. Moreover, it follows from the compactness of $[0,1]$, that $\mathcal{B}^{\cup}$ separates points off closed sets. It is a consequence of Proposition 3, that for each $\eta>0$ and for each gauge $\Delta$ there exists a $\left(\Delta, \eta, \mathcal{B}^{\cup}\right)$-Henstock partition. 
In order to exclude such pathologies we will assume - from now till the end of this section - that for each gauge $\Delta$ and each positive $\eta$ there exists a $(\Delta, \eta, \mathcal{B})$-Henstock (resp. McShane) partition of $\Omega$. Sometimes we will assume even more, that $\mathcal{B}$ is well founded for Henstock (resp. McShane) partitions, i.e.

If $W \subseteq \Omega$ is such that $\mu^{*}(W)=1$, then for each $\eta>0$ and each gauge $\Delta$ there exists a $(\Delta, \eta, \mathcal{B})$-Henstock (resp. McShane) partition tagged in $W$.

At the end of the paper we give two sufficient conditions on $\mathcal{B}$ such that the previous assumption is satisfied.

Now we can prove the following Cauchy condition for $\mathcal{B}$-integrals:

Proposition 2. $f$ is $\mathcal{B}_{H}$ (resp. $\mathcal{B}_{M S}$ ) -integrable on $\Omega$ if and only if the following Cauchy condition holds:

for each $\varepsilon>0$ there exist a gauge $\Delta$ and a positive constant $\eta$ such that

$$
\|\sigma(f, \mathcal{P})-\sigma(f, \mathcal{Q})\|<\varepsilon,
$$

for each couple $\mathcal{P}, \mathcal{Q}$ of $(\Delta, \eta, \mathcal{B})$-Henstock (resp. McShane) partitions.

Proof. Let $f$ be $\mathcal{B}_{H}$-integrable on $\Omega$ and let $w=\left(\mathcal{B}_{H}\right) \int_{\Omega} f d \mu$. Then, for each $\varepsilon>0$, there exists a gauge $\Delta$ and a positive constant $\eta$ such that

$$
\|\sigma(f, \mathcal{P})-w\|<\frac{\varepsilon}{2}
$$

for any $(\Delta, \eta, \mathcal{B})$-Henstock partition $\mathcal{P}$. Thus, if $\mathcal{P}$ and $\mathcal{Q}$ are two $(\Delta, \eta, \mathcal{B})$ Henstock partitions, we have

$$
\|\sigma(f, \mathcal{P})-\sigma(f, \mathcal{Q})\| \leq\|\sigma(f, \mathcal{P})-w\|+\|\sigma(f, \mathcal{Q})-w\|<\varepsilon .
$$

Now assume that the above Cauchy condition holds true and take $\varepsilon=1 / n$, for $n=1,2, \ldots$. There exists a gauge $\Delta_{n}$ and a positive constant $\eta_{n}$ such that

$$
\|\sigma(f, \mathcal{P})-\sigma(f, \mathcal{Q})\|<\frac{1}{n},
$$

for any couple $\mathcal{P}, \mathcal{Q}$ of $\left(\Delta, \eta_{n}, \mathcal{B}\right)$-Henstock partitions. We can assume $\Delta_{n}(\omega) \subseteq$ $\Delta_{n-1}(\omega)$, for each $\omega \in \Omega$ and $\eta_{n} \leq \eta_{n-1}$. Let $\mathcal{P}_{n}$ be a $\left(\Delta_{n}, \eta_{n}, \mathcal{B}\right)$-Henstock partition. Note that $\mathcal{P}_{m}$ is also a $\left(\Delta_{n}, \eta_{n}, \mathcal{B}\right)$-Henstock partition, if $m>n$. Then

$$
\left\|\sigma\left(f, \mathcal{P}_{n}\right)-\sigma\left(f, \mathcal{P}_{m}\right)\right\|<\frac{1}{n}, \text { if } m>n .
$$

Hence $\left\{\sigma\left(f, \mathcal{P}_{n}\right)\right\}$ is a Cauchy sequence in $X$. Let $w=\lim _{n} \sigma\left(f, \mathcal{P}_{n}\right)$. We claim that $w$ is the $\mathcal{B}_{H}$-integral of $f$ on $\Omega$. Indeed, for each $\varepsilon>0$ there exists $n$ 
such that $1 / n<\varepsilon$. Define $\Delta=\Delta_{n}$ and $\eta=\eta_{n}$. Let $\mathcal{P}$ be a $(\Delta, \eta, \mathcal{B})$-Henstock partition. Since, for each $m>n, \mathcal{P}_{m}$ is also a $(\Delta, \eta, \mathcal{B})$-Henstock partition, we have

$$
\left\|\sigma(f, \mathcal{P})-\sigma\left(f, \mathcal{P}_{m}\right)\right\|<\frac{1}{n}<\varepsilon .
$$

Thus

$$
\|\sigma(f, \mathcal{P})-w\|=\lim _{m}\left\|\sigma(f, \mathcal{P})-\sigma\left(f, \mathcal{P}_{m}\right)\right\| \leq \frac{1}{n}<\varepsilon .
$$

This completes the proof.

In our further investigations we will need one more definition:

Definition 3. Given a gauge $\Delta$, we say that $\Omega$ is $\Delta_{H}$-saturated by $\mathcal{B}$ if for each finite $\Delta$-fine family $\left\{\left(B_{1}, \omega_{1}\right), \ldots,\left(B_{n}, \omega_{n}\right)\right\}$ with all $\omega_{i} \in B_{i} \in \mathcal{B}$ and for each $\eta>0$ there exists a $(\Delta, \eta, \mathcal{B})$-Henstock partition of $\left(\bigcup_{i=1}^{n} B_{i}\right)^{c}$. In case $\Omega$ is $\Delta_{H}$-saturated by $\mathcal{B}$ for each gauge $\Delta$, then we say that $\mathcal{B}$ saturates $\Omega$ for Henstock partitions. In a similar way we define $\Delta_{M S}$-saturation.

At the end of the paper we give two sufficient conditions on $\mathcal{B}$ such that $\mathcal{B}$ saturates $\Omega$.

Theorem 5. Assume that $\mathcal{B}$ saturates $\Omega$ for Henstock partitions. If $f$ is $\mathcal{B}_{H^{-}}$ integrable on $\Omega$, then it is $\mathcal{B}_{H}$-integrable on $E$, for each measurable set $E \subset \Omega$. A similar result holds true for $\mathcal{B}_{M S}$-integral.

Proof. By Proposition 2, it is enough to prove that for each $\varepsilon>0$ there exists a gauge $\widetilde{\Delta}$ and a positive constant $\widetilde{\eta}$ such that

$$
\left\|\sigma\left(f \chi_{E}, \mathcal{P}\right)-\sigma\left(f \chi_{E}, \mathcal{Q}\right)\right\|<\varepsilon
$$

for any couple $\mathcal{P}, \mathcal{Q}$ of $(\widetilde{\Delta}, \widetilde{\eta}, \mathcal{B})$-Henstock partitions.

We fix $\varepsilon>0, \Delta$ and $\eta$ such that condition (10) holds for any couple $\mathcal{P}, \mathcal{Q}$ of $(\Delta, \eta, \mathcal{B})$-Henstock partitions. Let $F$ be a closed set and $G$ be an open set such that

$$
F \subset E \subset G \quad \text { and } \quad \mu(G \backslash F)<\eta / 4 .
$$

Define the gauge $\widetilde{\Delta}$ by setting

$$
\widetilde{\Delta}(\omega)= \begin{cases}\Delta(\omega) \cap G & \text { if } \omega \in F \\ \Delta(\omega) \cap(G \backslash F) & \text { if } \omega \in G \backslash F \\ \Delta(\omega) \cap F^{c} & \text { if } \omega \notin G .\end{cases}
$$


Given two $(\widetilde{\Delta}, \eta / 8, \mathcal{B})$-Henstock partitions $\mathcal{P}$ and $\mathcal{Q}$, we denote by $\mathcal{P}_{E}$ and $\mathcal{Q}_{E}$ the subpartitions tagged in $E$.

It follows directly from the definition of $\widetilde{\Delta}$ that

$$
\operatorname{co}\left(\mathcal{P}_{E}\right) \cap F=\operatorname{co}(\mathcal{P}) \cap F
$$

and

$$
\operatorname{co}\left(\mathcal{Q}_{E}\right) \cap F=\operatorname{co}(\mathcal{Q}) \cap F .
$$

Thus

$$
\left[\operatorname{co}\left(\mathcal{P}_{E}\right) \triangle \operatorname{co}\left(\mathcal{Q}_{E}\right)\right] \cap F=[\operatorname{co}(\mathcal{P}) \triangle \operatorname{co}(\mathcal{Q})] \cap F
$$

and

$$
\operatorname{co}\left(\mathcal{P}_{E}\right) \triangle \operatorname{co}\left(\mathcal{Q}_{E}\right)=\left[\operatorname{co}\left(\mathcal{P}_{E}\right) \triangle \operatorname{co}\left(\mathcal{Q}_{E}\right)\right] \cap F \cup\left[c o\left(\mathcal{P}_{E}\right) \triangle \operatorname{co}\left(\mathcal{Q}_{E}\right)\right] \cap(G \backslash F) .
$$

Consequently

$$
\mu\left[\operatorname{co}\left(\mathcal{P}_{E}\right) \triangle \operatorname{co}\left(\mathcal{Q}_{E}\right)\right]<\eta / 4+\eta / 4=\eta / 2 .
$$

Since $\mathcal{B}$ is $\widetilde{\Delta}_{H}$-saturated, there exists a $\widetilde{\Delta}$-fine $\mathcal{B}$-Henstock partition $\mathcal{O}$ in $\Omega \backslash\left[\operatorname{co}\left(\mathcal{P}_{E}\right) \cup \operatorname{co}\left(\mathcal{Q}_{E}\right)\right]$ such that $\mu\left(\Omega \backslash\left[\operatorname{co}\left(\mathcal{P}_{E}\right) \cup \operatorname{co}\left(\mathcal{Q}_{E}\right) \cup \operatorname{co}(\mathcal{O})\right]\right)<\eta / 2$. Then

$$
\mathcal{O} \cup \mathcal{P}_{E} \text { and } \mathcal{O} \cup \mathcal{Q}_{E}
$$

are two $(\Delta, \eta, \mathcal{B})$-Henstock partitions. Thus, by (10), we get (11) with $\widetilde{\eta}=$ $\eta / 8$.

Theorem 6. Assume that $\mathcal{B}$ is well founded and saturates $\Omega$ for Henstock partitions. If $f: \Omega \rightarrow \mathbf{R}$ is $\mathcal{B}_{H}$-integrable, then it is $\mu$-measurable. A similar result holds true for $\mathcal{B}_{M S}$-integral.

Proof. Suppose that $f$ is not $\mu$-measurable. Then there are reals $\alpha<\beta$ and $V \in \Sigma$ of positive measure, such that

$$
\mu^{*}(A)=\mu^{*}(B)=\mu(V)
$$

where

$$
A=\{\omega \in V: f(\omega) \leq \alpha\} \quad \text { and } \quad B=\{\omega \in V: f(\omega) \geq \beta\} .
$$

According to Theorem 5, the function $f \chi_{V}$ is $\mathcal{B}_{H}$-integrable. Now put $\varepsilon=\frac{1}{4} \mu(V)(\beta-\alpha)$ and let $\Delta: \Omega \rightarrow \mathcal{T}$ and $0<\eta<\frac{1}{4} \mu(V) \frac{\beta-\alpha}{|\alpha|+|\beta|}$ be such 
that (2) is satisfied by $f \chi_{V}$. Let $F_{1} \subseteq V$ and $F_{2} \subseteq V^{c}$ be closed sets such that $\mu\left[\left(F_{1} \cup F_{2}\right)^{c}\right]<\eta / 2$. Then define a new gauge by setting

$$
\tilde{\Delta}(\omega)= \begin{cases}\Delta(\omega) \cap F_{2}^{c} & \text { if } \omega \in F_{1} \\ \Delta(\omega) \cap\left(F_{1} \cup F_{2}\right)^{c} & \text { if } \omega \notin F_{1} \cup F_{2} \\ \Delta(\omega) \cap F_{1}^{c} & \text { if } \omega \in F_{2} .\end{cases}
$$

The condition (2) is clearly satisfied also for $\tilde{\Delta}$ and $\eta$.

By the assumption $\mathcal{B}$ is well founded for Henstock partitions and so there exists a $(\tilde{\Delta}, \eta, \mathcal{B})$-Henstock partition $\mathcal{P}=\left\{\left(P_{i}, p_{i}\right): i \leq p\right\}$ of $\Omega$ tagged in $A \cup V^{c}$ and a $(\tilde{\Delta}, \eta, \mathcal{B})$-Henstock partition $\mathcal{Q}=\left\{\left(Q_{j}, q_{j}\right): j \leq q\right\}$ of $\Omega$ tagged in $B \cup V^{c}$. Suppose, that

$$
\mathcal{P}_{A}=\left\{\left(P_{i}, p_{i}\right): i \leq p_{0} \leq p\right\}=\left\{\left(P_{s}, p_{s}\right): p_{s} \in A\right\}
$$

and

$$
\mathcal{Q}_{B}=\left\{\left(Q_{j}, q_{j}\right): j \leq q_{0} \leq q\right\}=\left\{\left(Q_{r}, q_{r}\right): q_{r} \in B\right\} .
$$

It follows, that

$$
\left|\left(\mathcal{B}_{H}\right) \int_{V} f d \mu-\sum_{i=1}^{p_{0}} f\left(p_{i}\right) \mu\left(P_{i}\right)\right|<\varepsilon
$$

and

$$
\left|\left(\mathcal{B}_{H}\right) \int_{V} f d \mu-\sum_{j=1}^{q_{0}} f\left(q_{j}\right) \mu\left(Q_{j}\right)\right|<\varepsilon .
$$

Hence

$$
\left(\mathcal{B}_{H}\right) \int_{V} f d \mu<\alpha \mu\left(\mathcal{P}_{A}\right)+\varepsilon \quad \text { and } \quad\left(\mathcal{B}_{H}\right) \int_{V} f d \mu>\beta \mu\left(\mathcal{Q}_{B}\right)-\varepsilon
$$

and so

$$
\beta \mu\left(\mathcal{Q}_{B}\right)-\varepsilon<\alpha \mu\left(\mathcal{P}_{A}\right)+\varepsilon
$$

Let

$$
a=\mu(V)-\mu\left(\mathcal{P}_{A}\right) \quad \text { and } \quad b=\mu(V)-\mu\left(\mathcal{Q}_{B}\right) .
$$

It follows from the properties of the sets $F_{1}$ and $F_{2}$, that

$$
\mu(V)-\mu\left(\mathcal{P}_{A}\right)=\mu\left(V \backslash F_{1}\right)+\mu\left(F_{1}\right)-\mu\left(\mathcal{P}_{A} \cap F_{1}\right)-\mu\left[\mathcal{P}_{A} \cap\left(F_{1} \cup F_{2}\right)^{c}\right] \leq \frac{3}{2} \eta
$$

because $\left(V \backslash F_{1}\right) \cup \mathcal{P}_{A} \cap\left(F_{1} \cup F_{2}\right)^{c} \subseteq\left(F_{1} \cup F_{2}\right)^{c}$ and $\mathcal{P}$ is a $(\tilde{\Delta}, \eta, \mathcal{B})$-Henstock partition. 
In a similar way, we get the inequality

$$
\mu\left(\mathcal{P}_{A}\right)-\mu(V)=\left[\mu\left(\mathcal{P}_{A} \backslash F_{1}\right)-\mu\left(V \backslash F_{1}\right)\right]+\left[\mu\left(\mathcal{P}_{A} \cap F_{1}\right)-\mu\left(F_{1}\right)\right]<\frac{3}{2} \eta .
$$

By analogy

$$
\left|\mu(V)-\mu\left(\mathcal{Q}_{B}\right)\right|<\frac{3}{2} \eta .
$$

Then we have

$$
\begin{aligned}
& \frac{1}{2} \mu(V)(\beta-\alpha)=2 \varepsilon>\beta \mu\left(\mathcal{Q}_{B}\right)-\alpha \mu\left(\mathcal{P}_{A}\right) \\
& =(\beta-\alpha) \mu(V)-b \beta+a \alpha \\
& \geq(\beta-\alpha) \mu(V)-|b||\beta|-|a||\alpha| \\
& >(\beta-\alpha) \mu(V)-\frac{3}{2} \eta(|\alpha|+|\beta|) \\
& >\frac{5}{8}(\beta-\alpha) \mu(V)
\end{aligned}
$$

which is impossible.

Remark 3. In the case of an arbitrary Banach space valued function $f: \Omega \rightarrow$ $X$ the $\mathcal{B}$-integrability of $f$ may be insufficient for measurability of $\|f\|$. To see it consider a function $f:[0,1] \rightarrow l_{2}[0,1]$ defined by

$$
f(t)=\left\{\begin{array}{cc}
e_{t} & \text { if } t \in V \\
0 & \text { if } t \notin V,
\end{array}\right.
$$

where $V$ is the Vitali set and $\left\{e_{t}: t \in[0,1]\right\}$ is the ordinary orthonormal basis of $l_{2}[0,1] . f$ is McShane integrable, but $\|f(t)\|=\chi_{V}(t)$ is non-measurable.

Corollary 1. Assume that $\mathcal{B}$ is well founded and saturates $\Omega$ for Henstock partitions. If $f:[0,1] \rightarrow \mathbf{R}$ is $\mathcal{B}_{H}$ (resp. $\mathcal{B}_{M S}$ ) -integrable, then $|f|$ is $\mathcal{B}_{H}$ $\left(\right.$ resp. $\left.\mathcal{B}_{M S}\right)$-integrable.

Proof. By Theorem 6 the set $E=\{x: f(x)>0\}$ is measurable. Then the functions $f \chi_{E}$ and $f \chi_{E^{c}}$ are $\mathcal{B}$-integrable, by Theorem 5 . Hence $|f|$ is $\mathcal{B}$-integrable, since $|f|=f \chi_{E}-f \chi_{E^{c}}$.

Theorem 7. Assume that $\mathcal{B}$ is well founded and saturates $\Omega$ for Henstock partitions. Then $f: \Omega \rightarrow \mathbf{R}$ is $\mathcal{B}_{H}$ (resp. $\mathcal{B}_{M S}$ ) -integrable on $\Omega$ if and only if it is Lebesgue integrable. Moreover, the integrals coincide. 
Proof. Suppose $f$ is Lebesgue integrable. Then it is Fremlin integrable [2] and so it is also $\mathcal{B}$-integrable and the integrals coincide.

Now suppose that $f$ is $\mathcal{B}$-integrable. Let $E=\{\omega: f(\omega) \geq 0\}$. According to Theorem $6, E$ is measurable. Then by Theorem $5, f \chi_{E}$ is $\mathcal{B}$-integrable. Now, if $g: E \rightarrow[0, \infty)$ is a Lebesgue integrable function, such that $g \leq f \chi_{E}$, then

$$
(L) \int_{E} g d \mu=(\mathcal{B}) \int_{E} g d \mu \leq(\mathcal{B}) \int_{E} f d \mu<+\infty .
$$

It follows, that $(L) \int_{E} f d \mu$ exists. In a similar way we get the existence of (L) $\int_{\Omega \backslash E} f d \mu$. Consequently, $f$ is Lebesgue integrable, and so, $(L) \int f d \mu=$ (B) $\int f d \mu$.

Theorem 8. Assume that $\mathcal{B}$ is well founded and saturates $\Omega$ for Henstock partitions. If $f: \Omega \rightarrow X$ is $\mathcal{B}_{H}$ (resp. $\mathcal{B}_{M S}$ ) -integrable, then it is Pettis integrable.

Proof. Given a measurable set $E \subset \Omega, f$ is $\mathcal{B}$-integrable on $E$, by Theorem 5. Let $w_{E}=(\mathcal{B}) \int_{E} f d \mu$ and let $x^{*} \in X^{*}$.

By the $\mathcal{B}$-integrability of $f$ on $E$, for each $\varepsilon>0$ there exist $\Delta$ and $\eta$ such that

$$
\left\|\sigma\left(f \chi_{E}, \mathcal{P}\right)-w_{E}\right\|<\varepsilon,
$$

for any $(\Delta, \eta, \mathcal{B})$-partition $\mathcal{P}$. Then

$$
\begin{aligned}
& \left|\sigma\left(x^{*}\left(f \chi_{E}\right), \mathcal{P}\right)-x^{*}\left(w_{E}\right)\right| \\
& \leq\left\|x^{*}\right\|\left\|\sigma\left(f \chi_{E}, \mathcal{P}\right)-w_{E}\right\|<\varepsilon\left\|x^{*}\right\| .
\end{aligned}
$$

This implies that $x^{*} f$ is $\mathcal{B}$-integrable on $E$ and $(\mathcal{B}) \int_{E} x^{*} f d \mu=x^{*}\left(w_{E}\right)$. By Theorem 7 , it is Lebesgue integrable on $E$ with $(L) \int_{E} x^{*} f d \mu=x^{*}\left(w_{E}\right)$. Therefore $f$ is Pettis integrable.

In fact the following more general result holds true: If $f: \Omega \rightarrow X$ is $\mathcal{B}_{H}$ (resp. $\mathcal{B}_{M S}$ )-integrable, $Y$ is a Banach space and $T: X \rightarrow Y$ is a bounded linear operator, then $T f: \Omega \rightarrow Y$ is $\mathcal{B}_{H}\left(\mathcal{B}_{M S}\right)$-integrable $T\left[\left(\mathcal{B}_{H}\right) \int f\right]=$ $\left(\mathcal{B}_{H}\right) \int T f \quad\left(\right.$ resp. $\left.T\left[\left(\mathcal{B}_{M S}\right) \int f\right]=\left(\mathcal{B}_{M S}\right) \int T f\right)$.

Since each strongly measurable and Pettis integrable function is Fremlin integrable [2] we get the following:

Theorem 9. Assume that $\mathcal{B}$ is well founded and saturates $\Omega$ for Henstock partitions. Let $f: \Omega \rightarrow X$ be a strongly measurable function. Then $f$ is $\mathcal{B}_{H}$ (resp. $\mathcal{B}_{M S}$ ) -integrable iff $f$ is Pettis integrable. 


\section{Existence of $(\Delta, \eta, \mathcal{B})$-Partitions.}

Let us denote by $\mathcal{T}_{\partial}$ the family of all open sets $G$ such that $\mu(\partial G)=0$. Then we have the following

Proposition 3. Let $\mathcal{B} \subseteq \Sigma$ be a fine family such that $\mathcal{T}$ is $\mathcal{B}^{\cup}$-inner regular and $\mathcal{T}$ is such that given $U \in \mathcal{T}$ and $\omega \in U$, there exists $V \in \mathcal{T}_{\partial}$ such that $\omega \in V \subseteq U$. Then $\mathcal{B}^{\cup}$ is well founded for Henstock partitions. Moreover, for each gauge $\Delta: \Omega \rightarrow \mathcal{T}_{\partial}$ the family $\mathcal{B}^{\cup}$ saturates $\Omega$ for Henstock partitions.

Proof. Let $\eta>0$ and a gauge $\Delta: \Omega \rightarrow \mathcal{T}$ be given. Then $\Omega \subseteq \bigcup_{\omega \in \Omega} \Delta(\omega)$. By the hypothesis, we may assume $\mu(\partial \Delta(\omega))=0$, for each $\omega \in \Omega$.

Proceeding as in the proof of Lemma 1 we find a finite collection $\bar{\omega}_{1}, \ldots, \bar{\omega}_{n} \in$ $W$ such that

(i) $\bar{\omega}_{i} \notin \overline{\Delta\left(\bar{\omega}_{j}\right)}$, for $j<i$;

(ii) $\mu\left(\Omega \backslash \bigcup_{i=1}^{n} \overline{\Delta\left(\bar{\omega}_{i}\right)}\right)<\eta / 2$.

Since $\mathcal{B}^{\cup}$ separates points off closed sets, there exists $B_{1} \in \mathcal{B}^{\cup}$ such that $\bar{\omega}_{1} \in B_{1} \subset \Delta\left(\bar{\omega}_{1}\right)$ and $\left.\mu\left(\overline{\Delta\left(\bar{\omega}_{1}\right)} \backslash B_{1}\right)\right)<\eta / 2 n$.

Similarly there exists $B_{2} \in \mathcal{B}^{\cup}$ such that $\bar{\omega}_{2} \in B_{2} \subset\left(\overline{\Delta\left(\bar{\omega}_{1}\right)}\right)^{c} \cap \Delta\left(\bar{\omega}_{2}\right)$ and $\left.\mu\left(\left[\left(\overline{\Delta\left(\bar{\omega}_{1}\right)}\right)^{c} \cap \Delta\left(\bar{\omega}_{2}\right)\right] \backslash B_{2}\right)\right)<\eta / 2 n$. At the step $j, 1<j \leq n$ there exists $B_{j} \in \mathcal{B}^{\cup}$ such that $\bar{\omega}_{j} \in B_{j} \subset\left(\bigcup_{s<j} \overline{\Delta\left(\bar{\omega}_{s}\right)}\right)^{c} \cap \Delta\left(\bar{\omega}_{j}\right)$ and $\left.\mu\left(\left[\left(\bigcup_{s<j} \overline{\Delta\left(\bar{\omega}_{s}\right)}\right)^{c} \cap \Delta\left(\bar{\omega}_{j}\right)\right] \backslash B_{j}\right)\right)<\eta / 2 n$. Then

$$
\begin{aligned}
& \mu\left(\Omega \backslash \bigcup_{j=1}^{n} B_{j}\right) \\
& \leq \mu\left(\Omega \backslash \bigcap_{j=1}^{n} \overline{\Delta\left(\bar{\omega}_{j}\right)}\right)+\mu\left(\bigcup_{j=1}^{n} \overline{\Delta\left(\bar{\omega}_{j}\right)} \backslash \bigcup_{j=1}^{n} B_{j}\right) \\
& \left.\leq \mu\left(\Omega \backslash \bigcup_{j=1}^{n} \overline{\Delta\left(\bar{\omega}_{j}\right)}\right)+\sum_{j=1}^{n} \mu\left(\left[\left(\bigcup_{s<j} \overline{\Delta\left(\bar{\omega}_{s}\right)}\right)^{c} \cap \Delta\left(\bar{\omega}_{j}\right)\right] \backslash B_{j}\right)\right) \\
& <\frac{\eta}{2}+\frac{\eta}{2}=\eta,
\end{aligned}
$$

and $\left\{\left(B_{j}, \bar{\omega}_{j}\right): j \leq n\right\}$ is a $\left(\Delta, \eta, \mathcal{B}^{\cup}\right)$-Henstock partition tagged in $W$.

To see whether $\mathcal{B}^{\cup}$ saturates $\Omega$ for each gauge $\Delta: \Omega \rightarrow \mathcal{T}_{\partial}$ notice that if $\left\{\left(B_{1}, \omega_{1}\right), \ldots,\left(B_{n}, \omega_{n}\right)\right\}$ is a $\Delta$-fine family with $\Delta: \Omega \rightarrow \mathcal{T}_{\partial}$ and all $\omega_{i} \in B_{i} \in$ 
$\mathcal{B}^{\cup}$, then $\left(\bigcup_{i=1}^{n} B_{i}\right)^{c} \supseteq \Omega \backslash \bigcup_{i=1}^{n} \overline{\Delta\left(\bar{\omega}_{i}\right)}$ and the last set is open. Hence we can apply the first part of the proof to the set $\Omega \backslash \bigcup_{i=1}^{n} \overline{\Delta\left(\bar{\omega}_{i}\right)}$.

We also have:

Proposition 4. Let $\mathcal{B} \subseteq \Sigma$ be a family such that $\mathcal{B}$ separates points off closed sets. We assume moreover that $\mu(\bar{B} \backslash B)=0$ for each $B \in \mathcal{B}$. Then $\mathcal{B}$ is well founded for Henstock partitions. Moreover, $\mathcal{B}$ saturates $\Omega$ for Henstock partitions.

Proof. Let $\eta>0$ and a gauge $\Delta: \Omega \rightarrow \mathcal{T}$ be given. Proceeding as in the proof of Lemma 1 we find a finite collection $\left\{\bar{\omega}_{1}, \ldots, \bar{\omega}_{n}\right\} \subset W$ such that

(i) $\mu\left(\Delta\left(\bar{\omega}_{i}\right)\right)>0$ for each $i \leq n$;

(ii) $\bar{\omega}_{i} \notin \Delta\left(\bar{\omega}_{j}\right)$ for all $j<i \leq n$;

(iii) $a:=\mu\left(\Omega \backslash \bigcup_{i=1}^{n} \Delta\left(\bar{\omega}_{i}\right)\right)<\eta$.

By the outer regularity of $\mu$ and because $\mathcal{T}$ is Hausdorff, there exist pairwise disjoint open sets $V_{i}, i \leq n$, such that

$$
\bar{\omega}_{i} \in V_{i} \subseteq \Delta\left(\bar{\omega}_{i}\right) \quad \text { and } \quad \mu\left(V_{i}\right)<\frac{\eta-a}{2 n} \quad \text { for each } \quad i \leq n
$$

Then, by the inner regularity of $\mu$ and because $\mathcal{T}$ is Hausdorff, we can find for each $i \leq n$ a closed set $F_{i} \subset V_{i}$ with $\bar{\omega}_{i} \in F_{i}$. Now, since $\mathcal{B}$ separates points off closed sets, there exists $B_{1} \in \mathcal{B}$ such that

$$
\bar{\omega}_{1} \in B_{1} \subseteq \Delta\left(\bar{\omega}_{1}\right) \backslash \bigcup_{j \neq 1} F_{j}
$$

and

$$
\mu\left[\Delta\left(\bar{\omega}_{1}\right) \backslash \bar{B}_{1} \backslash \bigcup_{j \neq 1} F_{j}\right]<\frac{\eta-a}{2 n}
$$

Notice that

$$
\bar{B}_{1} \cap \bigcup_{j \neq 1}\left\{F_{j}\right\}=\emptyset .
$$

Assume now that we have already chosen pairwise disjoint sets $B_{1}, \ldots, B_{i} \in \mathcal{B}$ such that for each $j \leq i$

$$
\bar{\omega}_{j} \in B_{j} \subseteq \Delta\left(\bar{\omega}_{j}\right) \backslash \bigcup_{l<j} \bar{B}_{l} \backslash \bigcup_{l \neq j} F_{l}
$$


and

$$
\mu\left[\Delta\left(\bar{\omega}_{j}\right) \backslash \bigcup_{l \leq j} \bar{B}_{l} \backslash \bigcup_{l \neq j} F_{l}\right]<\frac{\eta-a}{2 n} .
$$

Then, since $\mathcal{B}$ separates points off closed sets, there exists $B_{i+1} \in \mathcal{B}$ such that

$$
\bar{\omega}_{i+1} \in B_{i+1} \subseteq \Delta\left(\bar{\omega}_{i+1}\right) \backslash \bigcup_{l<i+1} \bar{B}_{l} \backslash \bigcup_{l \neq i+1} F_{l}
$$

and

$$
\mu\left[\Delta\left(\bar{\omega}_{i+1}\right) \backslash \bigcup_{l \leq i+1} \bar{B}_{l} \backslash \bigcup_{l \neq i+1} F_{l}\right]<\frac{\eta-a}{2 n} .
$$

It follows that

$$
\begin{aligned}
& \mu\left(\bigcup_{i=1}^{n} \Delta\left(\bar{\omega}_{i}\right) \backslash \bigcup_{i=1}^{n} B_{i}\right) \leq \mu\left(\bigcup_{i=1}^{n} \Delta\left(\bar{\omega}_{i}\right) \backslash \bigcup_{i=1}^{n} \bar{B}_{i} \backslash \bigcup_{i=1}^{n} F_{i}\right)+\mu\left(\bigcup_{i=1}^{n} F_{i}\right) \\
& <\sum_{i=1}^{n} \mu\left[\Delta\left(\bar{\omega}_{i}\right) \backslash \bigcup_{j \leq i} \bar{B}_{j} \backslash \bigcup_{j \neq i} F_{j}\right]+\frac{\eta-a}{2}<\eta-a .
\end{aligned}
$$

Thus,

$$
\mu\left(\Omega \backslash \bigcup_{i=1}^{n} B_{i}\right) \leq \mu\left(\Omega \backslash \bigcup_{i=1}^{n} \Delta\left(\bar{\omega}_{i}\right)\right)+\mu\left(\bigcup_{i=1}^{n} \Delta\left(\bar{\omega}_{i}\right) \backslash \bigcup_{i=1}^{n} B_{i}\right)<\eta .
$$

The family $\left\{\left(B_{i}, \bar{\omega}_{i}\right): i \leq n\right\}$ is the required $(\Delta, \eta, \mathcal{B})$-Henstock partition.

\section{References}

[1] D. H. Fremlin, Topological Riesz spaces and measure theory, Cambridge Univ. Press, (1974).

[2] D. H. Fremlin, The generalized McShane integral, Illinois J. Math., 39(1995), 39-67.

[3] R. A. Gordon, The integrals of Lebesgue, Denjoy, Perron and Henstock, Graduate Studies in Matm., vol. 4(1994), AMS.

[4] E. Marczewski and R. Sikorski, Remarks on measure and category, Coll. Math., 2(1949), 13-19.

[5] E. J. McShane, Unified integration, Academic Press, San Diego, (1983). 